\title{
Identificaciones policiales por perfil racial. Estudio criminológico sobre las experiencias de identificación en España en 2020-2021
}

Police identifications according to racial profile. Criminological study on the experiences of identification in Spain in 2020-2021

\section{Identificações policiais por perfil racial. Estudo criminológico sobre as experiências de identificação na Espanha em 2020-2021}

\author{
Hassiba Ziati Ziati ${ }^{\mathrm{a},}{ }^{\mathrm{*}} \mid$ Ariadna Trespaderne Dedeu $^{\mathrm{b}} \mid$ Nassiba Ziati Ziati \\ Miguel Ángel Soria Verde ${ }^{d}$ \\ a https://orcid.org/0000-0003-1454-6277 Universitat de Barcelona, Barcelona, España \\ ${ }^{\mathrm{b}}$ https://orcid.org/0000-0002-5125-5770 Universitat de Barcelona, Barcelona, España \\ c https://orcid.org/0000-0001-8564-7062 Universitat de Barcelona, Barcelona, España \\ ${ }^{d}$ https://orcid.org/0000-0002-6165-0950 Universitat de Barcelona, Barcelona, España
}

- Fecha de recepción: 2021-08-29

- Fecha concepto de evaluación: 2021-10-07

- Fecha de aprobación: 2021-10-18

https://doi.org/10.22335/rlct.v14i1.1487
Para citar este artículo / To reference this article / Para citar este artigo: Ziati Ziati, $H_{\text {., }}$ Trespaderne Dedeu, A., Ziati Ziati, N., \& Soria Verde, M. Á. (2022). Identificaciones policiales por perfil racial. Estudio criminológico sobre las experiencias de identificación en España en 2020-2021. Revista Logos Ciencia \& Tecnología, 14(1), 41-56. https://doi.org/10.22335/rlct. v14i1.1487

\begin{abstract}
RESUMEN
Uno de los temas principales respecto a las identificaciones policiales, es el uso de la raza como criterio en la toma de decisiones durante las retenciones por parte de la policía. Sin embargo, estudios previos han constatado que estas prácticas se concentran en mayor proporción en ciertos colectivos minoritarios, aplicando por ello el perfil étnico. A pesar de ser una práctica extendida en España y Europa, existen pocos estudios sobre las disparidades raciales entre grupos con relación a las identificaciones policiales. Por ello, la presente investigación pretende analizar las diferencias entre la población general y los grupos minoritarios con base en las experiencias de identificación policial en España durante 2020-2021. Para ello, se ha seguido una metodología cuantitativa, donde se han reportado los datos de identificaciones policiales por medio de un cuestionario con una muestra de 134 participantes. El análisis de los datos se ha realizado mediante asociación y modelo de regresión binaria. Los resultados sugieren la existencia de diferencias significativas entre grupos, en relación con las experiencias de identificación policial, pues las minorías étnicas son colectivos que tienen más probabilidades de ser señalados en comparación con la población general.
\end{abstract}

Palabras clave: Perfil racial, identificaciones policiales, etnia, vía pública, vehículo 


\begin{abstract}
One of the main issues regarding police identifications is the use of race as a criterion in decision-making during arrests. However, previous studies have shown that these police practices are more concentrated in certain minority groups, thus applying the ethnic profile. Despite being a widespread practice in Spain and Europe, there are few studies on racial disparities between groups regarding police identifications. Therefore, this research aims to analyze the differences between the general population and minority groups based on the experiences of police identification in Spain during 2020-2021. To that purpose, a quantitative methodology has been followed, in which police identification data have been reported through a questionnaire, with a sample of 134 participants. Data analysis has been performed using association analysis and binary regression model. The results suggest the existence of significant differences between groups regarding the experiences of police identification, since ethnic minorities, are groups that are most likely to be singled out compared to the general population.
\end{abstract}

Keywords: Racial profile, police arrests, ethnicity, public highway, vehicle

\title{
RESUMO
}

Uma das principais questões relacionadas à identificaçãoes policiais é o uso da raça como critério na tomada de decisão durante as retenções por parte da polícia. No entanto, estudos anteriores revelaram que essas práticas policiais concentram-se em maior proporção, em determinados grupos minoritários, aplicando assim o perfil étnico. Apesar de ser uma prática generalizada na Espanha e na Europa, existem poucos estudos sobre as disparidades raciais entre grupos em relação às identificações policiais. Portanto, esta pesquisa tem como objetivo analisar as diferenças entre a população em geral e os grupos minoritários a partir de experiências de identificação policial na Espanha durante 2020-21. Para isso, seguiu-se uma metodologia quantitativa, onde os dados de identificação policial foram reportados por meio de um questionário com uma amostra de 134 participantes. A análise dos dados foi realizada por meio de associação e modelo de regressão binária. Os resultados sugerem a existência de diferenças significativas entre os grupos, em relação às experiências de identificação policial, sendo as minorias étnicas o grupo com maior probabilidade de ser identificado em relação à população em geral.

Palavras-chave: Perfil racial, identificações policiais, etnia, via pública, veículo

Las identificaciones policiales se definen como la potestad que tiene la policía para detener a alguien en la vía pública y solicitar los documentos de identificación. En términos de Bradford (2017), las paradas se conciben como aquellas interacciones entre la policía y la comunidad, donde el agente de policía para a una persona en el espacio público o semipúblico y lleva a cabo una serie de requerimientos como puede ser una identificación. Esta definición permite facilitar la comparación con prácticas análogas como el Stop and Search (parada y cacheo), en Reino Unido o el Stop and Frisk (parada y cacheo), en Estados Unidos (López-Riba, 2019).

En España esta actividad se encuentra legalmente amparada en virtud de los artículos 16 y 17 de la Ley Orgánica 4/2015 del 30 de marzo sobre protección de la seguridad ciudadana. Según el artículo 16 de la Ley Orgánica de Protección de la Seguridad Ciudadana, en adelante
LOPSC, un policía puede requerir la identificación de las personas en dos supuestos: a) cuando existan indicios de que han participado en la comisión de una infracción; y b) cuando, en atención a las circunstancias concurrentes, se considere razonable y necesario que una persona acredite su identidad para prevenir la comisión de un delito. Asimismo, con base en el artículo 17 de la LOPSC, la policía puede llevar a cabo identificaciones colectivas con los mismos fines, pero dirigidas a la prevención de delitos de especial gravedad, así como para el descubrimiento y detención de las personas que hubieran participado en la comisión del crimen. Por tanto, según estos artículos, las identificaciones deben realizarse con el fin de detectar y prevenir infracciones penales o administrativas, y para evitar la ejecución de actos delictivos.

De acuerdo con los datos publicados en los Anuarios estadísticos del Ministerio el Interior, entre 2008 y 2015 se 
realizaron un total de 61.884.090 identificaciones policiales. De hecho, los datos de identificaciones anuales oscilan entre los nueve y los seis millones. A pesar de la reducción de la tasa de identificaciones en los últimos años, y teniendo en cuenta las cifras con relación a la población española, las identificaciones tienen un carácter generalizado. Además, estos datos no nos proporcionan información sobre los motivos que precipitaron las identificaciones ni tampoco las características de las personas identificadas, como puede ser la nacionalidad, el sexo, la edad, la apariencia étnica, entre otras.

Estudios previos muestran evidencias de casos en los que se produce una mayor proporción de identificaciones hacia grupos minoritarios étnicos, respecto a la población general (FRA, 2010; García-Añón et al., 2013; García et al., 2016; Glaser, 2014a; Glaser, 2014b; Home Office, 2015; Home Office, 2017; Miller, 2013; Norris et al., 1992; NYCLU, 2019; Office of the Attorney General of the State of New York, 1999; Open Society Justice Initiative, 2009a). Esto podría ser suficiente para indicar que los policías, en sus prácticas policiales, se concentran en determinados colectivos y utilizan de forma sistemática el denominado perfil étnico (Glaser, 2014a). Precisamente, una de las cuestiones más destacadas en materia de investigación y política pública a la que se enfrentan los administradores e investigadores policiales es el uso de la raza como criterio en la toma de decisiones policiales durante las paradas (Engel et al., 2006).

Sin embargo, el perfil racial constituye una práctica poco estudiada en Europa y España (García-Añón et al., 2013; Open Society Justice Initiative, 2009a). Desde un punto de vista académico, la investigación se motiva precisamente por lo ya expuesto: existen escasos precedentes en España sobre las identificaciones por perfil racial, con lo cual se pretende dar relevancia a un fenómeno muy extendido, pero poco estudiado por la investigación española. Por esta razón, en el presente estudio resulta interesante ofrecer una primera aproximación sobre el análisis de las diferencias entre la población general y los grupos minoritarios étnicos con relación a las experiencias de identificación policial, y con el fin de contribuir a señalar la preocupación existente y examinar este tipo de prácticas policiales, ya que se encuentran en la intersección entre los esfuerzos del control de la delincuencia y las cuestiones de procedimiento, equidad y responsabilidad policial.
Racial profiling en el pretexto de las identificaciones policiales

El concepto racial profiling, traducido como perfil racial en las identificaciones, hace referencia al uso de generalizaciones basadas en la raza, el origen étnico y nacional o la religión para iniciar una acción policial. El establecimiento de perfiles raciales se puede realizar en distintos ámbitos: la actuación policial ordinaria, la lucha contra el terrorismo, la toma de decisiones en cuestiones relativas a la inmigración, el asilo o en el control de fronteras (García-Añón et al., 2013; Glaser, 2014a). Withrow (2004) señala que este enfoque contiene dos dimensiones con implicaciones directas sobre los estudios empíricos de los perfiles raciales:

- Discrecionalidad: son acciones iniciadas por la policía, destacadas por la cautela policial; en ocasiones se considera necesaria para mejorar los efectos de la actividad policial.

- Factores legales y extralegales: identifica los dos tipos de factores a observar para la averiguación de pruebas de perfiles, es decir, aquellos factores extralegales de los ciudadanos como la raza y los factores legales como el comportamiento de los ciudadanos.

Los defensores de la elaboración de perfiles étnicos, con el pretexto de hacer identificaciones policiales, afirman que esta práctica es una buena actuación policial para la prevención, detección e investigación de la delincuencia $y$, por ello, el desarrollo de tipos de perfiles es una estrategia policial aceptada y permitida siempre que los perfiles no crucen la línea que distingue la actuación policial y legítima de las prácticas discriminatorias (Open Society Justice Initiative, 2009a; Open Society Justice Initiative, 2009b). Por ejemplo, Miller (2013), en su estudio, sugiere que las paradas realizadas por la policía local tienen más probabilidades de tener un componente paratextual, puesto que es más propensa a incorporar la condición racial y la juventud como marcadores dignos de un elevado escrutinio.

Sin embargo, hay motivos legítimos para preocuparse por el establecimiento de perfiles raciales en materia de identificaciones y registros policiales. En primer lugar, la elaboración de perfiles raciales es, en gran medida, cognitiva, en el sentido de que refleja los pensamientos y juicios de los agentes del orden. Por tanto, si los juicios de los agentes no se relacionan de forma fiable con sus 
acciones, estas prácticas pueden generar una parte desproporcionada de sanciones en el sometimiento de esos grupos a detenciones, registros y arrestos ilícitos, así como la estigmatización de grupos enteros como comunidades sospechosas, que contribuyen a la sobrerrepresentación de las minorías étnicas en otras partes del sistema de justicia penal, perpetuando los estereotipos y legitimando el racismo, lo cual plantea dudas sobre el uso de esta facultad institucional (Glaser, 2014a; Open Society Justice Intitative 2009a; Quinton, 2011).

Paradas y registros en la vía pública

Análisis de los datos globales sobre identificaciones policiales

En la figura 1 puede observarse la evolución de las identificaciones policiales Ilevadas a cabo en España, Inglaterra, Gales y Nueva York entre 2008 y 2015. En primer lugar, es preciso tener en cuenta que no se dispone de datos accesibles sobre identificaciones a nivel nacional para el resto de los periodos. El número de identificaciones anuales en España está entre los nueve y los seis millones, mientras que en el Reino Unido el número de identificaciones anuales oscila entre los veintidós mil y el millón y medio, y en Nueva York se encuentra entre las veintidós mil y seis cientos mil identificaciones respectivamente (figura 1). Esta información solamente permite reflejar los datos a modo global y proviene de información oficial proporcionada por los distintos anuarios estadísticos del Ministerio del Interior (MIR, 2008; MIR, 2009; MIR, 2010; MIR, 2011; MIR, 2012; MIR, 2013; MIR, 2014 \& MIR, 2015), el Boletín anual estadístico sobre poderes y procedimientos policiales en Inglaterra y Gales (2017), y el Informe de Unión de Libertades Civiles de Nueva York (2019).

Figura 1

Personas identificadas por los Cuerpos de Seguridad del Estado

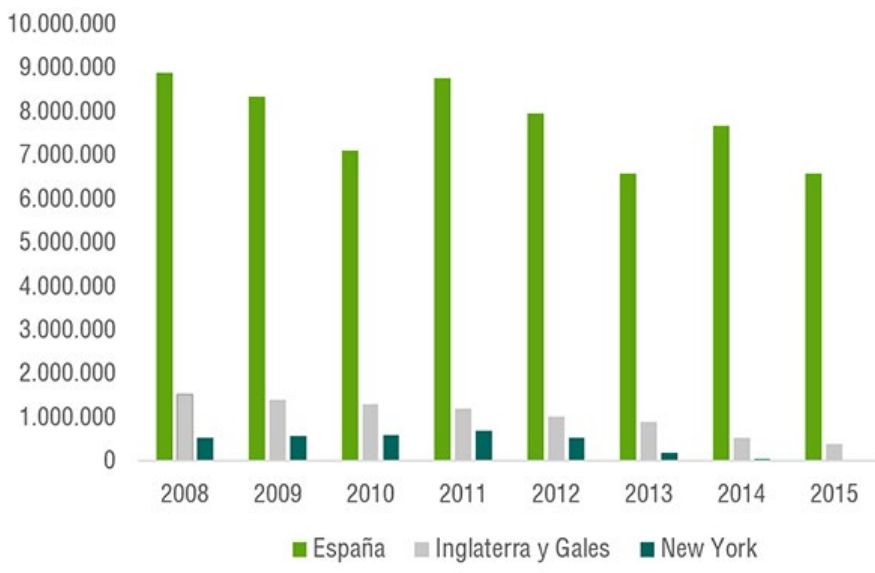

Nota: Elaboración propia a partir de los Anuarios Estadísticos del Ministerio del Interior, de NYCLU (2019) y de United Kingdom Government (2017).
Para saber si existe una tasa elevada de identificaciones, se puede establecer una comparación entre las identificaciones realizadas por la policía en España y las prácticas análogas de policías en otros contextos. Entre 2008 y 2015, España tuvo una tasa elevada de identificaciones por cada cien mil personas. Estas cifras son bastante altas si las comparamos con otros países como, por ejemplo, Reino Unido y Estados Unidos (Nueva York), los cuales presentaron tasas inferiores por cada cien mil habitantes durante los mismos periodos respectivamente (tabla 1 ).

Tabla 1

Tasas comparadas de identificaciones policiales por cada $100 \mathrm{mil}$ habitantes

\begin{tabular}{c|c|c|r}
\hline Año & España & Inglaterra y Gales & Nueva York \\
\hline 2008 & 19.447 .17 & 2.770 .81 & - \\
2009 & 18.038 .64 & 2.545 .44 & - \\
2010 & 15.277 .64 & 2.340 .60 & 3.099 .43 \\
2011 & 18.800 .93 & 2.118 .32 & $3.516,67$ \\
2012 & 16.998 .78 & 1.798 .80 & 2.721 .99 \\
2013 & 14.081 .63 & 1.587 .47 & 977.61 \\
2014 & 16.479 .02 & 942.62 & 233 \\
2015 & 14.171 .46 & 38.92 & 114.8 \\
\hline
\end{tabular}

Nota: Elaboración propia a partir de los Anuarios Estadísticos del Ministerio del Interior (2008-2015), INE (2020), NYCLU (2019), Office for National Statics (2020), United Kingdom Government (2017) y United States Census Bureau (2019).

Sin embargo, independientemente de la información más o menos detallada, estos resultados deben leerse con atención, puesto que existie disparidad en la exigencia la metodología de recolección y la publicación de los datos. En todo caso, esto indica que la policía en España utiliza las paradas policiales en mayor proporción respecto a otros países (López-Riba, 2019).

Datos por apariencia étnica o racial

Según el estudio realizado en España por García-Añón et al. (2013), el Ministerio del Interior mostró que entre 2011 y 2013 las identificaciones realizadas a ciudadanos nacionales representaban el $88.26 \%$ de la población y constituía el $45.45 \%$ de las personas identificadas; mientras que los extranjeros en su conjunto representaban el $11.74 \%$ de la población, y fueron el $54.56 \%$ de los identificados. En el caso de la apariencia étnica, los gitanos, magrebíes y afrolatinoamericanos fueron detenidos en mayor proporción en la vía pública, con un $60 \%, 45 \%$ y $39 \%$, respectivamente; es decir, la policía identificó 10 veces más a los gitanos, 7,5 veces más a los magrebíes y 6,5 veces más a los afrolatinoamericanos respecto al resto de la población. 
La encuesta EU-MIDIS realizada por la Agencia de Derechos Humanos en diciembre de $2009^{1}$ reveló que la tasa de personas retenidas entre inmigrantes y minorías étnicas fue superior respecto al total de la población. Si fueron parados mientras estaban en un vehículo, en el transporte público o en la calle, los resultados indicaron que entre el $70 \%$ y $98 \%$ de la mayoría de los encuestados se encontraban en un vehículo cuando fueron parados. Además, la policía también registró a los propios encuestados o a sus vehículos, con el 68\% de los romaníes en Grecia, el 46\% de los subsaharianos y el $38 \%$ de los norteafricanos en Francia y el 33\% de los norteafricanos en Bélgica (FRA, 2010).

Por otro lado, las últimas estadísticas publicadas sobre paradas y registros ${ }^{2}$ en Inglaterra y Gales muestran que, los miembros de grupos étnicos negros y minoritarios tenían el doble de probabilidades de ser retenidos y registrados que los blancos. Concretamente, las personas de raza negra tenían cuatro veces más probabilidades de ser detenidas que las de raza blanca (Home Office, 2015). En este sentido, el informe de la Inspección de la Policía de su Majestad (HMIC) (2013) examinó los registros policiales de las identificaciones realizadas y descubrió que: la mayoría de las fuerzas (33) registraron si una detención fue resultado de una parada o registro, y solo once de ellos registraron el motivo de la detención y el $27 \%$ no tenía pruebas suficientes y motivos razonables para realizar dicha retención.

En el caso de Nueva York, uno de los primeros análisis realizados fue el de la Oficina del Fiscal General del Estado de Nueva York (1999), y se enmarcó en la investigación Stop and Frisk de la Unidad de delincuencia Callejera de la policía de Nueva York. Tras un periodo de quince meses de estudio, la Fiscalía General descubrió que los ciudadanos negros y latinos fueron detenidos por la policía en proporciones mucho mayores que los blancos. Jones-Brown et al. (2010) analizaron los datos de las identificaciones del Departamento de Policía de Nueva York entre 2003 y 2009 y descubrieron un constante y marcado aumento en el número de identificaciones (de 160.851 en 2003 a 575.996 en 2009). Analizando en profundidad los datos de 2008, se reveló que el motivo más registrado para una detención era altamente subjetivo.

1 La encuesta se llevó a cabo entre los meses de mayo y noviembre del mismo año en los 10 Estados Miembros de la UE y con una muestra de 23.500 personas (FRA, 2010)

2 Abarcan los 12 meses hasta finales de marzo de 2015 (Home Office, 2015).
Por otra parte, según los datos proporcionados por el Informe de la Unión de Libertades Civiles de Nueva York [NYCLU] (2019), de las paradas registradas entre 2014 y 2017 el $53 \%$ fueron personas negras, $28 \%$ de latinas y un $11 \%$ de blancas. Con independencia de la composición del barrio y la disminución del número de identificaciones anuales, esto supone que la población negra y latina sigue siendo un objetivo desproporcionado por las prácticas de retención y cacheo de la policía de Nueva York.

Por tanto, se puede hacer una inferencia bastante fuerte sobre el sesgo racial, a partir de las tasas más altas de registros y en ausencia de las de arrestos para las minorías. Además, las estadísticas de registros y paradas indican que las decisiones de parar pueden basarse más en el comportamiento objetivo y la sospecha, mientras que las identificaciones policiales discrecionales, así como las decisiones de registro pueden estar más influidas por la raza y la etnia. Por ello, es más probable que los juicios sesgados por estereotipos se produzcan en situaciones ambiguas (Glaser, 2014b; Norris et al., 1992). Sin embargo, aunque las diferencias raciales en los índices de identificaciones y registros apuntan a la posibilidad de discriminación, las estadísticas oficiales sólo permiten tener un alcance limitado de la realidad puesto que presentan una imagen parcial y carecen de poder explicativo $y$, por tanto, no pueden aportar pruebas concretas de su existencia ya que no nos dicen nada sobre el origen y razones de estas disparidades (Bowling \& Philips, 2003).

\section{Eficacia de las paradas policiales por perfil racial: estudios previos}

\section{Eficacia indagatoria}

La falta de coherencia y proporcionalidad entre el número de personas identificadas y el número de personas detenidas con posterioridad se reflejan en informes $y$ encuestas como el estudio de la Agencia de Derechos Fundamentales, EU-MIDIS 2009, donde las minorías fueron interrogadas en mayor medida por la policía como consecuencia de la retención. Por ejemplo, en el caso de España, el $42 \%$ de los identificados eran norteafricanos, el $25 \%$, sudamericanos; el $18 \%$, rumanos; y solo el $12 \%$, nacionales españoles. Además, de todos ellos fueron detenidos $3.8 \%$ de norteafricanos; $2.2 \%$ de sudamericanos; $2.5 \%$ de rumanos, y $2.8 \%$ de españoles (FRA, 2010).

Los datos de la encuesta proporcionada por el estudio de García-Añón et al. (2013) muestran como la gran mayoría de las paradas en vía pública no tuvieron ningún tipo de 
resultado, es decir, ninguna actuación policial posterior $y$, por tanto, tales paradas no fueron acertadas ni efectivas. Por el contrario, las personas con nacionalidades diferentes a la española si tuvieron mayor probabilidad de ser arrestadas, detenidas o sometidas a cualquier otra actuación jurídico-penal (con un valor cercano al 10\%).

Un escenario similar surge con el estudio de García et al. (2016) extraído de la evaluación PIPE en las Policías Locales de Castellón y Pedrezuela. Según los resultados disponibles, el número de identificaciones positivas ${ }^{3}$ (126) representa el $28.9 \%$ del total de las practicadas; estos datos son parecidos tanto para españoles como extranjeros. La eficacia tiene que ver con las razones que las motivaron; para el caso de Castellón, la tasa de Pedrezuela es inferior. Según los resultados disponibles, la eficacia fue de un $22.6 \%$, con una mayor asociación entre extranjeros respecto a ciudadanos nacionales.

Pese a la información analizada, aunque las personas procedentes de grupos minoritarios tienen más probabilidades de ser paradas, no existe una diferencia criminológica o sancionadora significativa en cuanto al resultado de tales paradas, lo cual indica que la selección del perfil étnico constituye un criterio altamente inefectivo para identificar a personas que puedan haber cometido un delito o infracción ilegal (García-Añón et al., 2013). No obstante, estos datos son esenciales para poder identificar la posibilidad de cualquier uso desproporcionado de identificaciones contra determinadas comunidades.

\section{Eficacia preventiva}

Existen varias formas por las que las actuaciones de identificación y registro pueden provocar una reducción de la delincuencia. Por un lado, esta reducción se rige por medio de la detección directa de los delincuentes que han cometido o están a punto de cometer un delito. Por otro lado, con base en la perspectiva, las identificaciones policiales pueden disuadir a las personas paradas y a la población general, incrementando el riesgo percibido de detección directa y/o indirectamente, como parte de iniciativas más amplias con el fin de preservar el mantenimiento del orden (Miller et al., 2000).

Para evaluar los posibles efectos disuasorios, así como la relación existente entre el aumento de paradas policiales y la reducción de la criminalidad, se han llevado a cabo

3 Se entiende por identificaciones positivas todas aquellas que conllevan una denuncia administrativa o penal, o bien una requisitoria posterior, lo que indica que la identificación ha tenido un resultado posterior (García et al., 2016). investigaciones con datos observacionales agregados en diferentes unidades temporales y geográficas (LópezRiba, 2019). Rosenfeld y Fornango (2014) utilizaron los datos anuales desde 2003 a 2010 de paradas policiales con el fin de explorar los efectos semanales entre las paradas y los delitos, y concluyeron que aquellas no tenían efectos significativos sobre algunos delitos como los robos o los atracos. Por tanto, no se encontraron relaciones de peso para establecer un vínculo entre las retenciones policiales y la reducción de delitos.

Weisburd y Majmundar (2017) utilizaron datos inferiores agregados a niveles temporal y espacial, para explorar los efectos disuasorios de las paradas policiales concentradas en los puntos conflictivos de la ciudad de Nueva York. Los resultados mostraron que las paradas tenían un efecto significativo aunque modesto sobre la delincuencia, cuando esta se dirigía de forma intensiva a lugares de alta criminalidad.

Tiratelli et al. (2018) analizaron datos diarios y semanales de los distritos de Londres durante diez años y descubrieron que la tasa de paradas policiales no tenía efectos significativos sobre la reducción de delitos, concretamente en hurtos, delincuencia relacionada con vehículos y daños. Únicamente tenían efectos significativos sobre los delitos relacionados con drogas. Además, los propios autores advierten sobre la baja eficacia de las retenciones policiales, pues estas prácticas por sí solas no tienen que ver únicamente con la delincuencia, sino más bien con la convergencia de otros mecanismos más plausibles para el control de la criminalidad.

Finalmente, López-Riba (2019) realizó una primera aproximación a los efectos preventivos de las identificaciones policiales en España, para explicar el mecanismo causal entre la reducción o el incremento de los delitos por medio de estas actuaciones. El incremento de las identificaciones policiales no supuso la reducción en la tasa de delitos. No obstante, al introducir desfases en la tasa de identificaciones (controlando el efecto de las tasas actuales y las tasas de patrulla), los resultados revelaron una mayor predicción en referencia a los delitos contra la salud pública y un posible efecto disuasorio durante el segundo y tercer mes para los delitos contra la propiedad, en los tres meses posteriores respecto a la tasa general de delitos.

Sin embargo, el conjunto de estudios tanto longitudinales como experimentales o semi experimentales cuentan generalmente con problemas metodológicos para identificar 
la relación causal y valorar el impacto específico de las identificaciones policiales en la delincuencia (Bradford, 2017; Weisburd \& Majmundar, 2017). De hecho, la base de datos sobre los efectos de la identificación y el registro en la reducción de la delincuencia, ya sea a través de estos mecanismos específicos o de forma más general, es muy limitada (Miller et al., 2000).

\section{Fundamentación criminológica}

\section{Broken Windows Policing}

El uso de la detención y el registro se ha asociado con la Ilamada teoría de las ventanas rotas. La hipótesis de esta teoría sostiene que los signos de desorden atraen más desorden y delincuencia. Por consiguiente, la policía puede desempeñar un papel clave en la prevención de una espiral de decadencia centrada en los desórdenes menores y los delitos menos graves en zonas donde aún no han sido afectadas por la delincuencia grave (Miller et al., 2000; Wilson \& Kelling, 1982).

En consecuencia, la tesis aboga por respuestas policiales proactivas que permitan reforzar y mejorar esos controles sociales, especialmente en aquellas zonas donde se han debilitado aquellos registros informales antes de que se conviertan en el caldo de cultivo de la criminalidad a mayor escala. Por ello, la naturaleza de las intervenciones policiales de ventanas rotas varía desde el uso de tácticas informales de aplicación de la ley (como advertencias, desalojo) hasta formales o más intrusivas (como paradas, cacheos, arrestos, detenciones) (National Academies of Scienses et al., 2018; Weisburd et al., 2016). Del mismo modo, la reducción del control social informal debido a desventajas económicas y familiares requeriría enfoques más proactivos de control de la delincuencia para establecer el orden (Parker et al., 2010).

\section{Procedural justice Policing}

Una innovación organizativa más reciente con enfoque comunitario es la policía de justicia procesal. Esta estrategia señala cómo la policía interactúa de forma cotidiana con los ciudadanos para ofrecer espacios de decisión que se manifiestan en demostraciones de equidad y respeto policial sobre la dignidad de una persona (National Academies of Scienses et al., 2018). En este sentido, se presume que los procesos policiales justos, como pueden ser una detención o citación, son menos objetables para quienes experimentan los contactos policiales directamente en términos de legitimidad policial.
Por otro lado, la fuerte dependencia de paradas y registros puede reducir el respeto y el deseo de las personas de cumplir con la policía, puesto que los que se hallan en el extremo receptor pueden ver esas tácticas como injustas y/o pesadas (Gau \& Brunson, 2010). Incluso los casos de contactos frecuentes e indeseables con la policía pueden inducir a ciertos sectores de la población a creer que le desagradan a la policía (Brunson, 2007).

\section{Inmigración vs delincuencia}

Las teorías criminológicas más prosperas, que justifican la relación entre la inmigración y la delincuencia, son aquellas investigaciones centradas en la explicación de los factores sociales (Shaw \& McKay, 1969). La explicación a esta relación no se debe a una predisposición delictiva por parte del colectivo inmigrante, sino más bien por el debilitamiento de las organizaciones locales y de los controles informales fruto de la movilidad y heterogeneidad poblacional.

Asimismo, la perspectiva del condicionamiento social describe cómo los subgrupos sociales pueden vincularse a la actividad delictiva, creando estereotipos que guían la sospecha policial. La interiorización de creencias sobre la criminalidad de subgrupos que se transmite a través del racismo, de estereotipos raciales o de representaciones por parte de los medios genera, por parte de la policía, la adopción de perfiles inconscientes hacia determinados grupos demográficos específicos de la sociedad (Smith \& Alpert, 2007).

Sin embargo, la evolución y mejora de los planteamientos metodológicos para el estudio de la conexión entre ambos fenómenos ha revelado la existencia de una relación negativa entre la inmigración y la delincuencia; es decir, el aumento de la población inmigrante en países de Europa y Estados Unidos ha contribuido de forma positiva a la reducción de las tasas de criminalidad conocida oficialmente (Rosenfeld \& Messner, 2009).

\section{Objetivos e hipótesis}

El objetivo principal de la presente investigación se centra en analizar las diferencias entre la población general y las minorías étnicas sobre el fenómeno de las identificaciones policiales en España durante el periodo 2020-2021. Para el alcance de estos objetivos se prevén otros objetivos más precisos dirigidos a: 
- Identificar las diferencias existentes entre grupos étnicos con relación a las experiencias de identificación.

- Realizar un modelo de regresión para predecir la probabilidad de ser identificado en función de la etnia.

Respecto a la hipótesis de partida, se plantea que la actividad de identificaciones policiales en España se dirige de forma desproporcionada hacia los miembros de los grupos minoritarios. Asimismo, se proponen a continuación las siguientes hipótesis alternas:

$\mathrm{H}_{1}$ : La policía tiende a aplicar perfiles raciales, es decir, tiende a identificar en proporciones diferentes a los grupos minoritarios y mayoritarios.

$\mathrm{H}_{2}$ : Los grupos étnicos tienen más probabilidades de ser identificados en la vía pública que la población general.

\section{Metodología}

\section{Diseño}

La investigación se clasifica como exploratoria, descriptiva, inductiva y transversal, en un momento concreto y en un contexto como el español, en el cual el estudio del perfil racial, en el pretexto de las identificaciones policiales, es escaso tanto a nivel nacional como europeo (Guevara et al., 2020; Rojas, 2015).

La aplicación del formulario tuvo lugar entre el jueves 25 de marzo y el 25 de abril de 2021. Dicho estudio empleó un sistema metodológico experimental, caracterizado por un diseño con postest únicamente y grupo control caracterizado por la aplicación de un cuestionario como técnica cuantitativa, administrado vía online a través de la plataforma Google Docs, que se difundió en redes sociales con el fin de medir las variables de predicción y resultado en cada uno de los grupos al final de la aplicación (Manterola et al., 2001; Manterola et al., 2015). La toma de datos inicial se llevó a cabo el viernes 26 de marzo con una prueba piloto conformada por 10 personas y que posteriormente fue agregada a la muestra final.

\section{Muestra de estudio}

La investigación se enmarca en un muestreo probabilístico estratificado (empleando como criterio de estratificación la variable de la raza $\left.{ }^{4}\right)$, con criterios de aleatorización

4 Desde el enfoque de la etnicidad, la raza es una modalidad como el sexo, clase o nación históricamente articulada y, por tanto, necesariamente plural de inscripción/problematización de la diferencia debido a un origen común y/o similitudes manifestadas bajo las mimas condiciones ecológicas (Lamus, 2012; Rodero \& Herrera, 2000) simple. El número total de cuestionarios distribuidos fue de 140; si bien la muestra final quedó compuesta por 134 personas la criba se produjo seleccionando aquellos sujetos legitimados para ser identificados situados entre las edades de 18 a 57 años y, aplicando como criterios de exclusión aquellos sujetos menores de edad (17 años). Solamente se trataron datos del periodo 2020-21.

\section{Descripción de la muestra}

El grupo control se componía de 69 sujetos, 38 hombres y 31 mujeres, con edades entre los 18 y los 57 años, procedentes de España, y con apariencia caucásica europea y de nacionalidad española (76.1\%).

En cuanto al grupo experimental, este estaba formado por 65 sujetos, 47 hombres y 18 mujeres entre los 20 y 57 años, procedentes de Marruecos, Argelia, Senegal, Ecuador, Colombia, Bolivia y Pakistán, con apariencia no caucásica y sin nacionalidad española (23.8\%).

\section{Instrumento}

El instrumento utilizado para recopilar la información del presente estudio fue un cuestionario administrado vía online a través de la plataforma Google Docs; el enlace se difundió a través de las redes sociales Twitter, Facebook e Instagram, durante el periodo de análisis, incluyendo fines de semana y horarios distintos para captar las franjas temporales de mayor actividad por parte de los usuarios. En el cuestionario se emplearon preguntas cerradas con formulaciones basadas en respuestas dicotómicas, de elección múltiple, cerradas escalares, preguntas abiertas y con escalas de intensidad. Los 30 ítems del cuestionario se estructuraron en torno a tres fases: i) datos sociodemográficos (10 ítems); ii) experiencias personales en relación con las actuaciones policiales (14 ítems); y iii) otros contactos con la policía (6 ítems).

\section{Análisis estadístico}

Se analizaron 18 variables de tipo dicotómicas y multi-opción:

- Características de las personas identificadas: sexo, (hombre o mujer); edad, (18 a 29, 30 a 40 y más de 50 años); región de procedencia, (España o fuera de España); apariencia étnica (caucásica europea o no caucásica); nacionalidad, (española u otra); situación laboral, (empleado, desempleado, estudiante o empleado-estudiante); localidad, (Barcelona, Girona o Lleida); área de residencia, (urbana, semiurbana o rural).

- Características de las personas no identificadas: sexo, (hombre o mujer); edad, (18 a 29, 30 a 40 y más de 
50 años); región de procedencia, (España o fuera de España); apariencia étnica, (caucásica europea o no caucásica); nacionalidad (española u otra); situación laboral, (empleado, desempleado, estudiante o empleado-estudiante); localidad, (Barcelona, Girona o Lleida); área de residencia (urbana, semiurbana o rural).

- Identificación: identificación policial en vía pública (sí o no) e identificación policial en vehículo (sí o no).

Se ejecutaron tanto análisis univariantes para describir las variables estudiadas como bivariantes para comparar el número de participantes que reportaron la ejecución o no de una identificación policial, ya sea en vía pública o en vehículo en función de los datos sociodemográficos.

Los datos extraídos fueron analizados con el paquete estadístico SPSS Statics 25. En principio se aplicó la prueba Chi-Cuadrado y, posteriormente, se elaboró un análisis de regresión logística binaria con el fin de estimar la probabilidad de ser identificado en presencia de las variables predictoras (sexo, apariencia étnica y nacionalidad).

\section{IResultados}

\section{Descriptivos de la muestra}

Con respecto al tipo de identificación (tabla 2), la mayoría de los participantes $(n=132)$ que reportaron haber sido identificados por la policía en vía pública, 56\% eran hombres y $81.3 \%$ mujeres. De estos el $18.7 \%$ tenía una edad promedio de 26.97 años. Con relación a la región de procedencia de los identificados, el $70.7 \%$ son extranjeros y el $29.3 \%$ españoles. Asimismo, la mayoría tenía trabajo $50.7 \%$, mientras que el $29.3 \%$ estaban desempleados, el $12 \%$ eran estudiantes, y el $8 \%$ corresponde a estudiantes con empleo. En relación con la localidad, Barcelona ha resultado ser la ciudad más frecuente $76 \%$, seguida de Girona con $22.5 \%$ y, por último, Lleida con $1.3 \%$. Además, el $48.8 \%$ residían en áreas semiurbanas, el $46.3 \%$ en áreas urbanas y el $2.5 \%$ en áreas rurales. En la mayoría de los casos, los sujetos identificados tenían rasgos no caucásicos $72.5 \%$ en comparación con los caucásicos $27.5 \%$. La mayoría eran ciudadanos españoles $78.7 \%$ y un $21.3 \%$ extranjeros.

Respecto al $44 \%$ de participantes que informó no haber sido identificado en la vía pública, el 59.6\% eran mujeres y el $40.4 \%$ hombres, la edad promedio es de 23.60 años, respectivamente. Con relación a la región de procedencia, el $78.9 \%$ es de España y el $21.5 \%$ de otra región. La mayoría de estas personas eran estudiantes, $54.4 \%$; empelados, $24.7 \%$; estudiantes con empleo, $10.6 \%$; y desempleados, $7 \%$. En cuanto a la ubicación, Barcelona es la localidad más frecuente $75.4 \%$, seguido de Girona $19.3 \%$ y Lleida $3.5 \%$. Asimismo, el $47 \%$ residía en el área urbana, $43.9 \%$, en áreas semiurbanas y $3.8 \%$ en áreas rurales. En la mayoría de los casos, los participantes no identificados tenían rasgos caucásicos 75.4\% en comparación con los no caucásicos $24.6 \%$. Además, la mayoría de estos eran nacionales $76.5 \%$ y el $23.5 \%$ extranjeros.

En relación con las paradas en vehículos, la mayoría de los identificados tenía una edad promedio de 26.65 años. El $76.3 \%$ eran hombres y el $23.7 \%$ mujeres. Asimismo, la procedencia de la mayoría es del exterior $51.2 \%$ mientras el $48.8 \%$ son de origen español. Respecto a la situación laboral, el $47.5 \%$ son empleados, el $23.8 \%$ desempleados, $26.3 \%$ estudiantes y $12.5 \%$ estudiantes que trabajan. En relación con la localidad, los identificados residen en su mayoría en Barcelona $76.3 \%$, seguido de Girona $22.5 \%$ y Lleida $1.3 \%$. Del mismo modo, $48.8 \%$ residían en áreas semiurbanas, $46.3 \%$ en áreas urbanas y $2.5 \%$ en áreas rurales. En cuanto a la etnia, la mayoría presenta rasgos no caucásicos $72.5 \%$ y $27.5 \%$ caucásicos. Por último, $78.7 \%$ son nacionales y $21.3 \%$ extranjeros.

Con respecto a los sujetos no identificados (tabla 2), la mayoría tenían una edad media de 23.83 años. El $55.6 \%$ mujeres y $44.4 \%$ hombres. La mayoría procedía de España $55.6 \%$ y $44.4 \%$ de otra región. Asimismo, el $40.3 \%$ tenía empleo, el $29.9 \%$ eran estudiantes, $19.4 \%$ estaban desempleados y, por último, $10.4 \%$ eran estudiantes y empleados. En relación con la localidad, la mayoría procedían de Barcelona 75.9\%, Girona 20.4\% y Lleida $1.9 \%$. El $50 \%$ residía en un área semiurbana, $42.6 \%$ en un área urbana y $7.4 \%$ en zona rural. Respecto a la tipicidad étnica, la mayoría de los sujetos tenían rasgos caucásicos $53.7 \%$ y $46.3 \%$ no caucásicos. Además, $72.2 \%$ son nacionales y $27.8 \%$ extranjeros.

Sexo

Existe una relación significativa con el hecho de ser identificado por la policía en vía pública, con un tamaño del efecto moderado $\left(\chi^{2}=23.560 ; g l=1 ; p<0.05\right)$. Los hombres representan el $81.3 \%$ de la población identificada, mientras que las mujeres solamente un $18.7 \%(n=75)$. 
Tabla 2

Características sociodemográficas de los participantes identificados en vía pública o vehículos

\begin{tabular}{|c|c|c|c|c|}
\hline \multirow{2}{*}{ Variables } & \multicolumn{2}{|c|}{ Identificados } & \multicolumn{2}{|c|}{ No identificados } \\
\hline & Vía pública & Vehículos & Vía pública & Vehículos \\
\hline \multicolumn{5}{|l|}{ Sexo } \\
\hline Hombre & $61(81.3)$ & $61(76.3)$ & $23(40.4)$ & $24(44.4)$ \\
\hline Mujer & $14(18.7)$ & $19(23.7)$ & $34(59.6)$ & $30(556)$ \\
\hline Edad & $(M=26.9718 \pm 57)$ & $(M=26.6518 \pm 57)$ & $(M=23.6020 \pm 52)$ & $(M=23.8320 \pm 43)$ \\
\hline \multicolumn{5}{|l|}{ Región de procedencia } \\
\hline España & $22(29.3)$ & $39(48.8)$ & $45(78.9)$ & $30(55.6)$ \\
\hline Fuera de España & $53(70.7)$ & $41(51.2)$ & $12(21.05)$ & $24(44.4)$ \\
\hline \multicolumn{5}{|l|}{ Apariencia étnica } \\
\hline Caucásica europea & $7(9.3)$ & $22(27.5)$ & $43(75.4)$ & $29(53.7)$ \\
\hline No caucásica & $68(90.7)$ & $57(72.5)$ & $14(24.6)$ & $25(46.3)$ \\
\hline \multicolumn{5}{|l|}{ Nacionalidad } \\
\hline Española & $50(66.7)$ & $63(78.7)$ & $51(89.5)$ & $39(72.2)$ \\
\hline Otra & $25(33.33)$ & $17(21.3)$ & $6(10.5)$ & $15(27.8)$ \\
\hline \multicolumn{5}{|l|}{ Situación laboral } \\
\hline Empleado & $38(50.7)$ & $38(47.5)$ & $16(24.6)$ & $16(29.6)$ \\
\hline Desempleado & $22(29.3)$ & $19(23.8)$ & $7(7)$ & $7(13)$ \\
\hline Estudiante & $9(12)$ & $13(16.3)$ & $27(54.4)$ & $27(50)$ \\
\hline Empleado-estudiante & $6(8)$ & $10(12.5)$ & $4(14)$ & $4(7.4)$ \\
\hline \multicolumn{5}{|l|}{ Localidad } \\
\hline Barcelona & $57(76)$ & $61(76.3)$ & $43(75.4)$ & $41(75)$ \\
\hline Girona & $18(24)$ & $18(22.5)$ & $11(19.3)$ & $11(20.4)$ \\
\hline Lleida & - & $10(1.3)$ & $2(3.5)$ & $1(1.9)$ \\
\hline \multicolumn{5}{|l|}{ Área de residencia } \\
\hline Urbana & $32(42.7)$ & $39(46.3)$ & $27(47.4)$ & $23(42.6)$ \\
\hline Semiurbana & $41(54.7)$ & $37(48.8)$ & $25(43.9)$ & $27(50)$ \\
\hline Rural & $1(1.3)$ & $1(2.5)$ & $5(8.8)$ & $4(7.4)$ \\
\hline
\end{tabular}

Nota 1. $\mathrm{N}=132$ en vía pública ( $n=75$ identificados en vía pública y $n=57$ no fueron identificados en vía pública). Los participantes tienen una media de 25.51 años.

Nota 2. $\mathrm{N}=134$ en vehículos ( $n=80$ identificados en vehículo y $n=54$ no fueron identificados en vehículo). Los participantes tienen una edad media de 25.51 años.

Análisis de asociación entre las variables sociodemográficas e identificaciones policiales

Con relación a las identificaciones en vehículo, los análisis estadísticos muestran la existencia de diferencias significativas $\left(\chi^{2}=14.060 ; g l=1 ; p<0.05\right)$ entre el sexo y el hecho de ser identificado. Así pues, los hombres representan un $71.8 \%$ mientras que las mujeres representan un $38.8 \%$.

\section{Edad}

Los análisis estadísticos muestran la existencia de diferencias estadísticamente significativas respecto a la edad, con un tamaño del efecto bajo $\left(\chi^{2}=6.695 ; g l=2 ; p<0.05\right)$. El $84.9 \%$ de los jóvenes entre 18 y 29 años son identifi- cados en vía pública frente al $12.3 \%$ de los adultos entre los 30 y 40 años y al $2.3 \%$ con más de 50 años. En el caso de las identificaciones en vehículo, los estadísticos reflejan diferencias significativas con un tamaño del efecto bajo $\left(\chi^{2}=3.381 ; g l=2 ; p<0.05\right)$. En términos similares, los jóvenes representan el $87.2 \%$ de las identificaciones, mientras que los mayores entre 30 y 40 años corresponde al $10.3 \%$ y al $2.6 \%$ en mayores de 50 años.

\section{Apariencia étnica}

Respecto a la apariencia étnica, la identificación en vía pública se haya efectivamente en una relación signifi- 
cativa con un tamaño del efecto elevado $\left(\chi^{2}=60.145\right.$; $g l=1 ; p<0.05)$. Las personas con apariencia no caucásica representan un $9.3 \%$ de las identificaciones en vía pública, mientras que las personas con rasgos no caucásicos representan un $90.7 \%$. Asimismo, si desglosamos estos datos en función de la apariencia no caucásica, se destaca que el $100 \%$ de las personas de etnia gitana y sudafricana son identificadas en la vía pública, seguido del $75.7 \%$ de los magrebís y el $60 \%$ de latinos. En cuanto a las identificaciones en vehículo, los análisis estadísticos muestran diferencias significativas con un tamaño del efecto bajo $\left(\chi^{2}=9.390 ; g l=1 ; p<0.05\right)$. Las personas con rasgos no caucásicos representan un $69.9 \%$ de los identificados frente al $43.1 \%$ de los caucásicos. Si desglosamos estos datos en función de la apariencia no caucásica, encontramos que el $38.7 \%$ son magrebís, seguido del $11.3 \%$ de etnia gitana, el $8.8 \%$ son de sudafricanos y el $7.6 \%$ latinos.

\section{Nacionalidad}

En cuanto a la nacionalidad e identificación en vía pública, los estadísticos descriptivos indican la existencia de diferencias estadísticamente significativas con un tamaño del efecto bajo $\left(\chi^{2}=9.375 ; g l=1 ; p<0.05\right)$. Los españoles suponen el $66.7 \%$ de los identificados en vía pública, frente al $33.3 \%$ de los extranjeros. Sin embargo, en el caso de no ser identificado, los nacionales representan un $89.5 \%$ de los casos respecto al $10.5 \%$ de los extranjeros. No obstante, en el contexto de las identificaciones en vehículo, los estadísticos muestran diferencias significativas $\left(\chi^{2}=0.756 g /=1 p<0.05\right)$. En este caso, las personas con nacionalidad española representan un $78.8 \%$ de los identificados, en comparación con el $21.3 \%$ de extranjeros. En este sentido, el $72.2 \%$ de los españoles no fueron identificados por la policía, con respecto al $27.8 \%$ de los extranjeros (tabla 3 ).

Los resultados estadísticos muestran que en este modelo únicamente son predictores significativos sexo $(b=1.934$; Wald $\left.\chi^{2}(1)=12.263 ; p<0.01\right)$; etnia $(b=2.935$; Wald $\left.\chi^{2}(1)=18.244 ; \quad p<0.05\right) ; \quad y$ nacionalidad $(b=0.283$; Wald $\left.\chi^{2}(1)=0.182 ; p<0.05\right)$.

El estadístico R, cuadrado de Nagelkerke, es de 0.661 y, por tanto, las variables predictoras: sexo, etnia y nacionalidad incrementan la probabilidad de ser identificado por la policía en la vía pública. Asimismo, la prueba de Hosmer y Lmeshow ofrece un valor de 0.574 , es decir, indica una buena adaptación del modelo estimado.
Tabla 3

Características sociodemográficas en las identificaciones policiales Modelo de regresión

\begin{tabular}{|c|c|c|c|c|}
\hline \multirow{2}{*}{ Variables } & \multicolumn{2}{|c|}{$\begin{array}{l}\text { Identificados } \\
\text { vía pública }\end{array}$} & \multicolumn{2}{|c|}{$\begin{array}{l}\text { Identificados } \\
\text { en vehículos }\end{array}$} \\
\hline & n (\%) & $P$ & n (\%) & $p$ \\
\hline Sexo & & $0.000^{*}$ & & $0.000^{*}$ \\
\hline Hombre & $61(81.3)$ & & $61(71.8)$ & \\
\hline Mujer & $14(18.7)$ & & $19(38.8)$ & \\
\hline \multicolumn{5}{|l|}{ Edad } \\
\hline Entre $18-29$ & $62(84.9)$ & $0.035^{* * *}$ & $68(87.2)$ & 0.184 \\
\hline Entre $30-40$ & $9(12.3)$ & & $8(10.3)$ & \\
\hline Más de 50 & $2(2.7)$ & & $2(2.6)$ & \\
\hline Apariencia étnica & & $0.002^{* *}$ & & $0.002^{* *}$ \\
\hline Caucásica europea & $7(9.3)$ & & $22(43.1)$ & \\
\hline No caucásica & $68(90.7)$ & & $58(69.9)$ & \\
\hline Nacionalidad & & $0.000^{*}$ & & 0.385 \\
\hline Española & $50(66.7)$ & & $63(78.8)$ & \\
\hline Otra & $25(33.3)$ & & $17(21.3)$ & \\
\hline
\end{tabular}

Nota. ${ }^{* * *} p<0.05{ }^{* *} p<0.01{ }^{*} p<0.001$

Respecto a las variables en la ecuación (tabla 4), el estadístico B permite identificar que la variable con más aplicación al modelo es la etnia. Estos datos sugieren que las personas con rasgos no caucásicos tienen más probabilidades de ser identificadas en vía pública que los caucásicos (2.935 unidades). La segunda variable que aporta más variación al modelo es la de sexo; esto se refleja en que los hombres tienen más probabilidades de ser identificados en vía pública que las mujeres (1.934 unidades). Respecto a la variable nacionalidad, encontramos que los extranjeros tienen más probabilidades de ser identificados en la vía pública, a diferencia de los españoles (0.283 unidades).

La relación entre las variables estimada por medio del estadístico exponencial de (B) indica la fortaleza de la relación entre una variable independiente y una variable dependiente. Los valores de Exponencial de B superiores a 1 nos indican que el aumento de la variable independiente se asocia a un incremento de la probabilidad del suceso. Por el contrario, los valores inferiores a 1 nos indican que el incremento de la variable independiente se asocia a una disminución en la probabilidad del suceso.

En este caso, la categoría etnia revela que, por cada disminución de una unidad en el hecho de ser identificado 
en vía pública, aumenta 18.820 veces más la probabilidad de ser identificado, teniendo en cuenta el resto de las variables del modelo. La variable sexo indica que, por cada disminución de una unidad en ser identificado en la vía pública, la probabilidad de ser identificado aumenta 1.145 veces, manteniendo constantes el resto de las variables. Finalmente, la variable nacionalidad muestra que, por cada disminución de una unidad en el supuesto de ser identificado, la probabilidad de ser identificado en vía pública aumenta 1.327 veces más.

Tabla 4

Coeficientes de regresión de las variables sociodemográficas de las identificaciones policiales en vía pública

\begin{tabular}{|c|c|c|c|c|c|c|}
\hline Predictores & $\beta$ & SE $\beta$ & $\begin{array}{c}\text { Wald's } \\
\chi^{2}\end{array}$ & $d f$ & $P$ & $e^{\beta}$ \\
\hline Sexo & 1.934 & 0.5522 & 12.263 & 1 & 0.000 & 1.145 \\
\hline Etnia & 2.935 & 0.687 & 18.244 & 1 & 0.000 & 18.820 \\
\hline Nacionalidad & 0.283 & 0.662 & 0.182 & 1 & 0.003 & 1.327 \\
\hline Test & & & $\chi^{2}$ & $d f$ & $P$ & \\
\hline $\begin{array}{l}\text { Evaluación } \\
\text { general del } \\
\text { modelo }\end{array}$ & & & 101.218 & 1 & 0.001 & \\
\hline
\end{tabular}

Nota. $R^{2}$ Cox y Snell $=0.452 ; R^{2}$ de Nagelkerke=0.606; Porcentaje Glo$\mathrm{bal}=82.6 \%$

Según los datos de la tabla de clasificación, de un total de 132 encuestados, 66 han sido clasificados correctamente. El porcentaje de clasificaciones correctas del modelo de regresión logística es del $82.6 \%$ de los casos. Se encontraron en total 80 casos identificados por la policía en vía pública, lo cual representa un $60.6 \%$ de los sujetos estudiados. Así pues, la sensibilidad de la identificación en vía pública para detectar los casos en los que se identificó a un sujeto es del $82.5 \%$ y la especificidad de $82.7 \%$.

\section{】 Discusión}

Según la presente investigación, que analiza las diferencias entre los grupos mayoritarios y minoritarios sobre el fenómeno de las identificaciones policiales, se encuentra que los resultados sobre las diferencias existentes entre grupos, con respecto a las identificaciones policiales, han sido observados parcialmente en investigaciones previas ya reseñadas (FRA, 2010; García-Añón, 2013; García et al., 2016; Glaser, 2014a; Glaser, 2014b; Miller, 2013; NYCLU, 2019; Norris et al., 1992; Office of the Attorney General of the State of New York, 1999), sobre todo entre la nacionalidad y la etnia.
Los datos obtenidos por apariencia étnica señalan que las prácticas de identificación en vía pública afectan en mayor proporción a las minorías étnicas. Concretamente, las personas con rasgos no caucásicos $90.7 \%$ representan la mayoría de las identificaciones en vía pública, respecto de las personas con rasgos caucásicos 9.3\%. Del mismo modo, de acuerdo con el estudio de NYCLU (2019), los negros $53 \%$ y los latinos $28 \%$ fueron parados por la policía con mayor frecuencia en comparación con los blancos $11 \%$. Es más, el estudio de Office of the Attorney General of the State of New York (1999) detectó que el mayor índice de paradas a negros presentaba un menor índice de sospecha respecto a la población blanca que era parada y detenida con un mayor índice de sospecha. Asimismo, el estudio de García-Añón (2013) muestra una baja efectividad en las paradas realizadas, es decir, tras la identificación realizada a los grupos minoritarios, éstas no tuvieron actuaciones policiales posteriores. Esto supone que, en términos de NYCLU (2019), los grupos minoritarios son objetivos desproporcionados en materia de identificaciones y detenciones.

De manera más concreta, en función del grupo étnico, los datos confirman que las personas de etnia gitana, magrebíes, sudafricanos y latinos tienden a ser identificados con mayor frecuencia que la población general. Esto se encuentra en consonancia con los estudios de García-Añón et al. (2013), en los cuales, a nivel nacional, se observa una mayor prevalencia de paradas hacia los gitanos, seguidos de los magrebíes y los afro latinoamericanos $y$, a nivel europeo tal y como se puede destacar en el estudio de FRA (2010), los colectivos más identificados son los romaníes, seguidos de los subsaharianos y norteafricanos.

Los datos reportados en el estudio revelan que conductores de minorías étnicas son más identificados en vehículo. Sucede lo mismo con otros estudios (Glaser, 2014b; NYCLU, 2019), donde se observa un mayor registro en las paradas entre negros y latinos respeto de la población blanca. Paralelamente, el estudio de Jones-Brown et al., (2010), señala que el principal motivo de estas paradas se debe a criterios altamente subjetivos, es decir, en indicadores de raza más que de comportamientos sospechosos o delictivos. Si es así, de acuerdo con Withrow (2004) los agentes policiales estarían utilizando factores como la raza, es decir, perfiles raciales como estrategias policiales para llevar a cabo su trabajo rutinario.

En los resultados de este estudio se observa una relación positiva entre la edad y las identificaciones policiales. De 
forma similar ocurre en Miller (2013) donde la raza y la juventud pueden constituir firmes predictores estadísticos en cuanto al registro de las identificaciones.

Los resultados obtenidos en la investigación también señalan que los extranjeros tienen más probabilidades que los nacionales de ser identificados. Esto se encuentra en consonancia con los estudios previamente mencionados (García-Añón et al., 2013; García et al., 2016), donde se observa una mayor prevalencia entre los extranjeros y las identificaciones. De hecho, resulta contraproducente que los inmigrantes tengan una mayor probabilidad de ser identificados respecto los nacionales por varias razones. En primer lugar, en cuanto a estos datos, debemos recalcar dos aspectos importantes a debatir. Por un lado, las identificaciones se llevan cabo con el fin de prevenir o detener alguna infracción que se esté cometiendo. Por otro lado, estudios empíricos de la criminología reciente como el realizado por Rosenfeld y Messner (2009) reflejan una asociación baja entre la inmigración y la delincuencia, es decir, que los inmigrantes contribuyen a generar a las poblaciones occidentales menos delincuencia. Por tanto, teniendo en cuenta los fines con los que se llevan a cabo las identificaciones, así como los estudios que apoyan la relación negativa entre delincuencia e inmigración, la probabilidad de ser identificado siendo extranjero revierte de solidez empírica.

Asimismo, los datos reportan una mayor probabilidad de ser más identificado en grupos minoritarios que mayoritarios. De hecho, estos resultados son comparables con otras investigaciones que han estudiado estas prácticas entre colectivos (Norris et al., 1992; Home Office, 2015; Home Office, 2017) donde reportaron una mayor probabilidad de ser identificado entre la población negra, más que la blanca o que grupos minoritarios. Sucede algo parecido en la investigación de Open Society Justice Initiative (2009a), pues, reveló que los inmigrantes y los grupos minoritarios tenían más probabilidades de ser retenidos en comparación con la población mayoritaria. En estos estudios, coincide la falta de regulación y criterios objetivos en los procedimientos de identificación, frente a las fases posteriores. Esto se traduce en que las identificaciones realizadas por los agentes de la policía se llevan a cabo bajo un amplio margen de discrecionalidad.

Por último, cabría discutir si los datos reportados en la presente investigación son suficientes para justificar un aparente uso extendido de las identificaciones hacia los grupos minoritarios. Sobre todo, por tres razones. En primer lugar, puesto que representan el uso de un po- der coactivo por parte de la policía, que puede llegar a tensar los derechos como la libertad de movimiento o la intimidad (Ríos et al., 2015). En segundo lugar, porque se ha demostrado que, en materia de identificaciones y registros, los colectivos minoritarios son principalmente afectados por la elaboración de perfiles raciales (GarcíaAñón et al., 2013). Y por último, porque la investigación criminológica ha puesto énfasis en los efectos adversos que puede generar esta práctica sobre determinados grupos. Estos efectos se traducen en una mayor persecución policial hacia las minorías étnicas, una mayor desproporcionalidad en la aplicación de sanciones, así como la creación de estereotipos y estigmatización de los grupos afectados. Además, las identificaciones policiales pueden tener consecuencias negativas en términos de justicia procedimental y con ello, la reducción de la voluntad de los civiles de colaborar con la policía en un futuro (Gau \& Brunson, 2010).

\section{Conclusiones}

En conclusión, se puede afirmar la existencia de diferencias significativas entre grupos sobre las experiencias de identificación policial. Estas características difieren principalmente en función del sexo, la nacionalidad y la apariencia étnica. Los datos obtenidos en el presente trabajo revelan que los grupos minoritarios tienden a ser más identificados en vía pública respecto a la población general. En las identificaciones realizadas a vehículos, las diferencias entre grupos persisten, aunque no de forma tan predecible como el caso de la vía pública. Esto sugiere que las identificaciones en vehículos podrían producirse por la confluencia de otras características o comportamientos, además de los analizados. En el supuesto contrario, las personas no migrantes y los miembros de etnias mayoritarias son más propensas a no ser identificadas en la vía pública o en vehículo. Por tanto, se puede afirmar la existencia de un patrón de utilización de perfiles étnicos en los controles sistemáticos de identificación por parte de la policía en ambos contextos.

Si bien, parece que los resultados de la investigación se añaden a una literatura con resultados dispares, las diferencias entre grupos es más probable sobre el pretexto de las identificaciones policiales (ya sea a nivel correlacional o en términos de probabilidad). Así pues, tanto la nacionalidad como el sexo son factores que determinan que un individuo que no sea español tenga más probabilidades de ser identificado, pero, en relación con la apariencia étnica, la probabilidad aumenta mucho más. 
De esta manera, al igual que otros estudios, los datos de la investigación sustentan un mayor incremento en la tasa de identificaciones en los grupos minoritarios respecto a los mayoritarios.

En efecto, al igual que en otras investigaciones (Glaser, 2014a; Glaser, 2014b; Open Society Justice Intitative 2009a; Quinton, 2011; Smith \& Alpert, 2007) el incremento de las experiencias de identificación policial hacia determinadas minorías étnicas se debe en gran medida a la aplicación de perfiles raciales como consecuencia de la falta de criterios e indicadores objetivos capaces de justificar y legitimar las actuaciones policiales llevadas a cabo.

Finalmente, puesto que esta investigación es una primera aproximación a las variaciones entre grupos con relación a las experiencias de identificación policial, es necesario continuar con investigación que permita profundizar en el mecanismo causal de las disparidades raciales en las experiencias de identificación. Con este fin, es preciso considerar hasta qué punto los perfiles raciales realmente mejoran la seguridad pública, y con ello, considerar hasta qué punto se basan en diferencias reales y significativas en la criminalidad relativa, en contraposición a generalizaciones defectuosas.

\section{Referencias}

Bowling, B., \& Philips, C. (2003). Racism, ethnicity and criminology. Developing minority perspectives. British Journal of Criminology, 43(2), 269-290. https://doi. org/10.1093/bjc/43.2.269

Bradford, B. (2017). Stop and Search and Police Legitimacy. Routledge.

Brunson, R. K. (2007). Police don't like black people: African-American Young men's accumulated police experiences. Criminology \& Public Policy, 6(1), 71-102.

Engel, R., Calnon., \& Bernard, T. J. (2006). Theory racial profiling: Shortcomings and future directions in research. Justice Quarterly, 19(2), 249-273. https://doi. org/10.1080/07418820200095231

FRA (European Union Agency for Fundamental Rights). (2010). Data in Focus Resport 4: Police stops and minorities. EU-MIDIS. European Union Minorities and discrimination survey, European Union Agency for Fundamental Rights.

García-Añón, J., Bradford, B., García, S. J. A., Gascón, A., \& Llorente, A. (2013). Introducción: ¿En qué consiste la identificación por perfil étnico? En Identificación policial por perfil étnico en España (pp. 30-39). Tirant lo Blanch.

García, E., Arenas, L., \& Miller, J. (2016). Identificaciones policiales y discriminación racial en España. Evaluación de un programa para su reducción. Boletín Criminológico, 22(161-167), 1-9. https://doi.org/10.24310/ Boletin-criminologico.2016.v22i2016.7546

Gau, J. M., \& Brunson, R. K. (2010).Procedural Justice and Order Maintenance Policing: A study of Inner-City Young Men's Perceptions of Police Legitimacy. Justice Quarterly, 27(2), 255-279. https://doi. org/10.1080/07418820902763889

Glaser, J. (2014a). What Is Racial Profiling? En Suspect Race: Causes and Consequences of Racial Profiling (pp. 1-21). Oxford. http://doi.org/10.1093/acprof:o so/9780195370409.003.0002

Glaser, J. (2014b). Racial Profiling Is Real. En Suspect Race: Causes and Consequences of Racial Profiling (pp.1-22). Oxford. http://doi.org/10.1093/acprof:o so/9780195370409.003.0002

Guevara, P. G., Verdesoto, A. E., \& Castro, N. E. (2020). Metodologías de investigación educativa (descriptivas, experimentales, participativas, y de investigación-acción). Educational research methodologies (descriptive, experimental, particpatory, and action research). Recimundo, 4(3), 163-173. http://doi.org/10.26820/ recimundo/4.(3).julio.2020.163-173

HMIC (Inspecting policing in the public interest) (2013). Stop and Search Powers: Are the pólice using them effectively and fairly? http://www.justiceinspectorates.gov.uk/hmic/media/stop-and-searchpowers-20130709.pdf

Home Office. (2015). Police Powers and procedures England and Wales year ending 31 March 2015. Home Office.

Home Office. (2017). Police Powers and procedures England and Wales year ending 31 March 2017. Home Office.

INE. (2020). Población residente por fecha, sexo, grupo de edad y nacionalidad. https://www.ine.es/jaxiT3/ Datos.htm?t=9689

Jones-Brown, D., Gill. J., \& Trone, J. (2010). Stop, Question \& Frisk Policing Practices in New York City: A primer. John Jay College the city University of New York of Criminal Justice.

Lamus, D. (2012). Raza y etnia, sexo y género: El significado de la diferencia y el poder. Reflexión Política, 14(27), 68-84. 
Ley Orgánica 4/2015, de 30 de marzo, de protección de la seguridad ciudadana, BOE, núm. 77, de 31 de marzo de 2015 (2015). https://boe.es/buscar/act. php?id=BOE-A-2015-3442

López-Riba, J. M. (2019). ¿Tienen las identificaciones policiales efectos preventivos en la delincuencia? International e-Journal of Criminal Sciences, 8(14), 2-22.

Manterola, C., \& Bustos, L. (2001). Estrategias de investigación. Diseños experimentales. Ensayo clínico. Revista Chilena de Cirugía, 53(5), 498-503.

Manterola, C., \& Otzen, T. (2015). Estudios Experimentales $2^{\text {a }}$ Parte. Estudios Cuasi-Experimentales. International Journal of Morphology, 33(1), 382-387.

Miller, K. (2013). The Institutionalization of Racial Profiling Policy: An Examination of Antiprofiling Policy Adoption Among Large Law Enforcement Agencies. Crime \& Delinquency, 59(1), 32-58. https://doi. org/10.1177/0011128708328863

Miller, J., Bland, N., \& Quinton, P. (2000). The impact of stop and searches on crime and the community. Home Office.

MIR (Ministerio del Interior). (2008). Anuario Estadístico del Ministerio del Interior 2008. http://www.interior. gob.es/documents/642317/1204854/Anuario+estad\% C3\%ADstico+del+Ministerio+del+Interior+de+2008. pdf/2215b4dd-aeec-4430-8a0a-eb047d4cb056

MIR (Ministerio del Interior). (2009). Anuario Estadístico del Ministerio del Interior 2009. http://www.interior. gob.es/documents/642317/1204854/Anuario+estad\% C3\%ADstico+del+Ministerio+del+Interior+de+2009. pdf/fbec9f09-165a-4836-8c53-aec26c3eea23

MIR (Ministerio del Interior). (2010). Anuario Estadístico del Ministerio del Interior 2010. http://www.interior. gob.es/documents/642317/1204856/Anuario+Estad\% C3\%ADstico+del+Ministerio+del+Interior+2010.pdf/ b28597a1-c633-4cfa-b2c1-3f0094e55588

MIR (Ministerio del Interior). (2011). Anuario Estadístico del Ministerio del Interior 2011. http://www.interior.gob.es/documents/642317/1204756/Anuario+ estad\%C3\%ADstico+de+2011.pdf/1d35a1c8-f2e14417-bc5a-ca4e17bb7e66

MIR (Ministerio del Interior). (2012). Anuario Estadístico del Ministerio del Interior 2012. http://www.interior. gob.es/documents/642317/1204854/Anuario_estadistico_2012_okkk.pdf/00ee01c7-3122-496a-a023$22829653 \mathrm{e} 6 \mathrm{c} 0$

MIR (Ministerio del Interior). (2013). Anuario Estadístico del Ministerio del Interior 2013. http://www.interior.
gob.es/documents/642317/1204854/Anuario_Estadistico_2013.pdf/b7606306-4713-4909-a6e4-0f62daf29b5c

MIR (Ministerio del Interior). (2014). Anuario Estadístico del Ministerio del Interior 2014. http://www.interior. gob.es/documents/642317/1204854/Anuario-Estadistico-2014_v201510.pdf/0c18a800-f7f7-405c-9155$7391633618 \mathrm{c} 8$

MIR (Ministerio del Interior). (2015). Anuario Estadístico del Ministerio del Interior 2015. http://www. interior.gob.es/documents/642317/1204854/AnuarioEstadistico-2015.pdf/03be89e1-dd38-47a2-9ce8ccdd74659741

National Academies of Sciences, Engineering and Medicine. (2018). Proactive Policing: Effects on Crime and Comunities. National Academies Press. https://doi. org/10.17226/24928.

Norris, C., Fielding, N., Kemp, C., \& Fielding, J. (1992). Black and Blue: an analysis of the influence on being stopped by the police. The British Journal of Sociology, 43 (2), 207-224.

NYCLU. (2019). Anual Stop-and-Frisk in the Blasio Era. NYCLU.

Office for National Statics. (2020). Population estimates time series dataset. https://www.ons.gov.uk/peoplepopulationandcommunity/populationandmigration/ populationestimates/datasets/populationestimatestimeseriesdataset

Office of the Attorney General of the State of New York. (1999). The New York City Police Department's "Stop \& Frisk" Practices: A Report to the People of the State of New York From The Office Of The Attorney General. OAGSNY.

Open Society Justice Intitiative. (2009a). Adressing Ethnic Profiling by Police. A Report on the Strategies for Effective Police Stop and Search Project: Improving relations between police and minority communuties by increasing the fairness, effectiveness, and accountability of police stops in Bulgaria, Hungary, and Spain. Autor.

Open Society Justice Intitiative. (2009b). Ethnic Profiling in the European Union: Pervasive, Ineffective, and discriminatroy. Autor.

Parker, K. F., Lane, E. C., \& Alpert, G. P. (2010). Community characteristics and police search rates: Accounting for the ethnic diversity of urban areas in the study of Black, White, and Hispanic searches. En S. K. Rice \& M. D. White (eds.), Race, ethnicity, and policing: New and essential readings (pp.349-367). New York University Press. 
Quinton, P. (2011). The formation of suspicions: Police stop and serch practices in England and Wales. Policing and Society, 21(4), 357-368. https://doi.org/10.1 080/10439463.2011.610193

Rodero, E., \& Herrera, M. (2000). El concepto de raza. Un enfoque epistemológico. Archivos de Zootecnia, 49(186), 5-16.

Rojas, M. (2015). Tipos de investigación científica: una simplificación de la complicada incoherente nomenclatura y clasificación. Revista Electrónica de Veterinaria, 16(1), 1-14.

Rosenfeld, R., \& Fornango, R. (2014). The impact of police on precint robbery and burglary rates in New York City, 2003-2010, Justice Quarterly, 31(1), 96-122.

Rosenfeld, R., \& Messner, S. F. (2009). The crime in drop in comparative perspective: the impact of the economy and imprisonment on American and European burglary rates. The British Journal of Sociology, 60(3), 445-471.

Shaw, C. R., \& Mckay, H. D. (1969). Juvenile delinquency and urban areas (rev. ed.), University of Chicago Press.

Smith, M. R., \& Alpert, G. (2007). Explainig Police Bias. A Theory of Social Conditioning and illusory Correlation. Criminal Justice and Behavior. Criminal Justice and Behavior, 34(10). 1262-1283. https://doi. org/10.1177/0093854807304484
Tiratelli, M., Quinton, P., \& Bardford, B. (2018). Does Stop and Search deter crime? Evidence from ten years of London-wide data. British Journal of Criminology, 58, 1212-1231. http://doi:10.1093/bjc/azx085

United Kingdom Government. (2017). Police powers and procedures England and Wales year ending 31 March 2017 second edition. https://www.gov.uk/government/ statistics/police-powers-and-procedures-england-andwales-year-ending-31-march-2017

United States Census Bureau. (2019). State population totals and components of Change: 2010-2019. https:/www.census.gov/data/tables/time-series/demo/ popest/2010s-state-total.html

Withrow, B. (2004). A comparative Analysis of Commonly Used Benchmarks in Racial Profiling: A Research Note. Justice Reserach and Policy, 6(1), 71-92. https:// doi.org/10.3818/JRP.6.1.2004.71

Wilson, J. Q., \& Kelling, G. L. (1982). Broken windows: The police and neighborhoad safety. The Atlantic, March, 27-38.

Weisburd, D., \& Majmundar, M. K. (2017). Proactive Policing: Effects on crime and communities. The National Academy Press. 